\title{
A pain in the bud? Implications of cross-modal sensitivity for pain experience
}

\author{
Monica Perkins $^{1}$ - Marien de Bruyne ${ }^{2}$ - Melita J. Giummarra ${ }^{3,4}$
}

Published online: 14 October 2016

(C) The Psychonomic Society, Inc. 2016

\begin{abstract}
There is growing evidence that enhanced sensitivity to painful clinical procedures and chronic pain are related to greater sensitivity to other sensory inputs, such as bitter taste. We examined cross-modal sensitivities in two studies. Study 1 assessed associations between bitter taste sensitivity, pain tolerance, and fear of pain in 48 healthy young adults. Participants were classified as non-tasters, tasters and supertasters using a bitter taste test (6-n-propythiouracil; PROP). The latter group had significantly higher fear of pain (Fear of Pain Questionnaire) than tasters ( $p=.036$, effect size $\mathrm{r}=$ .48). There was only a trend for an association between bitter taste intensity ratings and intensity of pain at the point of pain tolerance in a cold pressor test ( $\mathrm{p}=.04)$. In Study 2, 40 healthy young adults completed the Adolescent/Adult Sensory Profile before rating intensity and unpleasantness of innocuous (33 $\left.{ }^{\circ} \mathrm{C}\right)$, moderate $\left(41^{\circ} \mathrm{C}\right)$, and high intensity $\left(44^{\circ} \mathrm{C}\right)$ thermal pain stimulations. The sensory-sensitivity subscale was positively correlated with both intensity and unpleasantness ratings. Canonical correlation showed that only sensitivity to audition and touch (not taste/smell) were associated with intensity of moderate and high (not innocuous) thermal stimuli. Together these findings suggest that there are cross-modal associations
\end{abstract}

Melita J. Giummarra

melita.giummarra@monash.edu

1 School of Psychological Sciences and Monash Institute of Cognitive \& Clinical Neurosciences, Monash University, Clayton, VIC, Australia

2 School of Biological Sciences, Monash University, Clayton, VIC, Australia

3 School of Public Health \& Preventive Medicine, Monash University, 99 Commercial Road Clayton, VIC Melbourne 3000, Australia

4 Caulfield Pain Management \& Research Centre, Caulfield, VIC Melbourne, Australia predominantly between sensitivity to exteroceptive inputs (i.e., taste, touch, sound) and the affective dimensions of pain, including noxious heat and intolerable cold pain, in healthy adults. These cross-modal sensitivities may arise due to greater psychological aversion to salient sensations, or from shared neural circuitry for processing disparate sensory modalities.

Keywords Pain $\cdot$ Taste $\cdot$ Fear $\cdot$ Cross-modal $\cdot$ Cold pressor test $\cdot$ Bitter sensitivity $\cdot$ PROP

Taste and pain are important sensory experiences for survival. Both generate automatic responses to avoid, withdraw, and reject aversive stimuli, acting as alerting signals to the presence of potentially threatening or toxic conditions. Noxious inputs are processed via two primary ascending neural pathways: the medial pathway and the lateral pathway. The medial pathway, via the medial thalamus, anterior cingulate cortex (ACC), amygdala, and insular cortex into regions in the frontal cortex, is predominantly involved in the processing of cognitive and emotional aspects of pain, including fear of pain (Kulkarni et al., 2005). The lateral pathway, on the contrary, processes sensory-discriminative aspects of the stimulus, such as intensity and the location of pain on the body. The affective ascending pathway fundamentally overlaps with neural regions that process other sensory phenomena, such as gustatory experiences, with overlap especially in the thalamus, ACC, amygdala, insular cortex, and orbitofrontal cortex (Small \& Apkarian, 2006). These regions are essentially involved in the generation of emotion, motivation, learning, and memory, and thus may play a key role in evaluating sensory experiences regardless of modality.

Several studies have investigated the association between sensitivity to pain and taste. Erden et al. (2007) established that increased bitter taste sensitivity was positively correlated 
with pain intensity when receiving an injection prior to surgery. While pleasant sweet taste elicits analgesia in neonates (Johnston et al., 2011), and may also reduce pain sensitivity in adult males (Priya, Siddanagoudar, Nalluwar, \& Neelam, 2015), adding bitterness to a sweet solution decreases analgesia (Eggleston, White, \& Sheehe, 2010), and removing an acutely painful stimulus (e.g., in a modified cold pressor test paradigm) seems to enhance perception of subsequent gustatory pleasure (Bastian, Jetten, \& Hornsey, 2014). Collectively, these findings suggest that there is a bidirectional valanced relationship between taste sensitivity or experience and pain, where stimulation in one domain (i.e., pleasant and unpleasant taste) influences experiences in another (i.e., with analgesia or hypoalgesia, respectively), and vice versa.

Sensitivity to bitter taste is partially driven by variation in the TAS2R38 gene on chromosome 5, which results in the inheritance of different levels of sensitivity to bitter tasting compounds (Duffy et al., 2004). Sensitivity to the compound propylthiouracil, commonly known as PROP, appears to vary partially in relation to these genetic variations, whereby one may be classified as extremely sensitive (super-taster), moderately sensitive (taster), or relatively non-sensitive (non-taster). People with chronic pain have enhanced sensitivity to a range of gustatory stimuli, including PROP (Small \& Apkarian, 2006). This may be due to the fact that people with acquired chronic pain have heightened activity in the insular, prefrontal, and cingulate cortex, with increased sensitivity to sensations processed via these neural networks including those that underscore bitter taste sensitivity (Formaker \& Frank, 2000; Small, 2010).

To date, however, it is not clear whether taste sensitivity and pain sensitivity (of pain-related fear) are strongly associated in healthy young adults. In particular, those who are more fearful of pain tend to avoid exposure to potentially painful experiences, and avoid activities that may exacerbate chronic pain (McNeil \& Rainwater, 1998; Vogt, 2005). On the contrary, persons who are more sensitive to PROP are more reactve to emotional stimuli, resulting in heightened approach/avoidance behaviours (Herbert et al., 2014; Macht \& Mueller, 2007).Fear of pain may therefore also be associated with the tendency to have a lower sensory threshold, and to avoid strong or noxious sensations.

In the present research, we undertook two studies, the first to examine the association between pain experience in the cold pressor test (CPT), fear of pain, and bitter taste sensitivity. The CPT involves immersion of the hand in ice-cold water and is frequently used as a test of cardiovascular and autonomic function; however, it has also been demonstrated to be a well validated experimental pain induction technique (Koenig et al., 2014). The CPT elicits an enormous sympathetic response that is also unpleasant, making it an ideal human model of clinical pain. As an experimental technique we typically quantify the time to pain onset (threshold), unbearable pain (tolerance) and perceived intensity and unpleasantness of the experience. We hypothesized that sensitivity to PROP would be positively correlated with perceived pain intensity and unpleasantness, and negatively correlated with pain tolerance time, in the CPT. Given that the neural regions for processing the affective dimensions of pain and bitter taste seem to overlap, we also hypothesized that greater bitter taste sensitivity would be associated with greater fear of pain.

As Study 1 only found that bitter taste sensitivity was associated with the intensity of cold pressor pain (but not with tolerance time, unpleasantness, or fear of pain), we undertook a second study to examine the association between pain experience and self-reported sensory preferences and sensitivity across a range of sensations, including taste, touch, movement, and vision. We administered the Adult/Adolescent Sensory Profile to measure self-reported sensory preferences (i.e., avoidance or seeking) and sensitivity (i.e., high sensitivity or low registration). Noxious thermal stimuli were administered to determine heat pain threshold, and subjective intensity and unpleasantness for a range of thermal stimuli. We hypothesized that high sensory sensitivity and avoidance of sensory stimuli would be associated with lower thermal pain threshold, and intensity and unpleasantness of moderate to high intensity thermal stimuli. Finally we explored whether self-reported sensitivity to specific sensory modalities (taste/ smell, touch, or audition) were associated with sensitivity to the thermal stimuli (i.e., intensity ratings of innocuous, moderate, and high intensity). These are the first studies to examine cross-modal sensitivities using acute experimental pain models in healthy young adults, and the research therefore provides preliminary evidence for the shared basis of sensory sensitivity while eliminating mechanisms that arise only in chronic pain conditions, like central sensitization.

\section{Study one}

\section{Materials and methods}

\section{Participants}

Forty-eight healthy individuals (57.4\% female) aged $18-34$ years ( $m=22.46, s d=3.50$ ), volunteered by responding to advertisements at Monash University. Previous studies have found that in a healthy undergraduate cohort, approximately $31.5 \%$ of participants will be super-tasters, $45.7 \%$ will be tasters, and $22.8 \%$ will be non-tasters (Herz, 2011). We aimed to test 50 participants to ensure that we would have at least 10-15 participants in each taster range to allow us to make meaningful comparisons between groups, and along the spectrum of bitter taste sensitivity.

Individuals with olfactory or gustatory dysfunctions, pain, or central nervous system disorders, or who were currently taking any medication (apart from the contraceptive pill in women) were excluded from participating. Written informed consent was obtained prior to commencing the experiment, and 
participants received AUD\$15 for their time. Ethics approval was obtained through the university human ethics committee.

\section{Measures}

Participants completed questionnaires about demographic information (e.g., age, sex, education), and the Fear of Pain Questionnaire (FOP; McNeil \& Rainwater, 1998). This measures enduring beliefs and behaviors relating to fear of painful experiences and procedures. It is weighted equally on a 5point Likert-scale $(0=$ not at all fearful, $5=$ extremely fearful $)$ and summed to provide a total score, with a range of 30-150. The index has demonstrated good test-retest reliability (Roelofs, Peters, Deutz, Spijker, \& Vlaeyen, 2005), and within the current sample, it had excellent internal consistency (Cronbach's $\alpha=.94)$.

PROP (6-n-propythiouracil) papers were made in accordance with previous research studies (Bartoshuk, et al., 1994; Small \& Apkarian, 2006). Five grams of PROP was added to $500 \mathrm{ml}$ of de-ionized water to make a saturated solution. The solution was then heated to near boiling to enhance dispersion, and $0.4 \mathrm{ml}$ (containing $4 \mu \mathrm{g}$ of $6-\mathrm{n}$ propythiouracil) was applied to each filter paper using a pipette. The papers were dried and stored in individual snaplock bags, and kept in the testing laboratory, which was maintained at a consistent temperature of approximately $22^{\circ} \mathrm{C}$ until required. The PROP papers were all used within 6 months of preparation.

The cold pressor apparatus consisted of a portable cooler filled with tap water to a depth of $20 \mathrm{~cm}$. Ice was added until the temperature reached $2{ }^{\circ} \mathrm{C}$. The water was constantly circulated with an internal pump to maintain the temperature at 2 $\circ$. The temperature of the water was recorded before, during and after hand immersion, and more ice was added if needed to maintain the target temperature.

\section{Procedure}

Participants were instructed not to consume caffeine $2 \mathrm{~h}$ prior to testing nor alcohol on the day of testing. Participants attended the laboratory to complete the questionnaire, CPT, and taste task. The order of the CPT and taste task was randomized in order to reduce the potential for acute pain to influence taste sensitivity and vice versa (e.g., see Bastian, Jetten, \& Hornsey, 2014).

During the CPT, participants were instructed to fully submerge their hand, with the hand in a flat position and the fingers extended, in the water until they felt they could no longer tolerate it (pain tolerance, recorded in seconds), at which point they could remove their hand. An upper limit of 5 min of submersion was enforced as 4-8\% of individuals are reported to adapt to the numbing sensation of the cold water, without recording pain (Wolff, 1984). Only two participants met this threshold. Participants rated the intensity and unpleasantness using a $10-\mathrm{cm}$ visual analogue scale (VAS) where the far left represented no sensation/not unpleasant $(=0)$ and extreme pain/extremely unpleasant at the far right $(=10)$.

To test sensitivity to bitter taste, participants were presented with the PROP filter paper and informed it contained a taste. Participants were instructed to place the entire paper onto their tongue and allow it to moisten with their saliva. Participants were then asked to rate the intensity of the taste at its maximum perceived intensity, by drawing a line on a general-labelled magnitude scale (g-LMS; Green, et al., 1996), which is consistent with previous studies investigating bitter taste sensitivity (e.g., Herz, 2011; Small \& Apkarian, 2006). The g-LMS is a semantic scale of perceptual intensity characterized by quasi-logarithmic spacing of its verbal labels, yielding ratio-level data.

The g-LMS and the VAS comprised 100 units and 10 units, respectively. The ratings were measured with a ruler to obtain the value corresponding to the point marked by the participant. Participants were classified as non-tasters (rating of 0 $15)$, tasters (16-49), and super-tasters (50-100), based on the values recommended by Herz (2011).

\section{Data analysis}

All data were analyzed in SPSS, version 23. Outliers were identified using a cut-off score of \pm 3.29 of corresponding $\mathrm{z}$ scores and adjusted to the next highest or lowest score (Tabachnick \& Fidell, 2013). Intensity ratings at pain tolerance and FOP were not normally distributed so we conducted non-parametric analyses, including Spearman's Rank-order correlations ( $\alpha=.05$ ), the Kruskal-Wallis test, and MannWhitney $U$ tests. These analyses examined the associations between bitter taste intensity with pain tolerance time, intensity, unpleasantness, and FOP total scores, and whether there were differences in pain experiences between groups (i.e., non-tasters, tasters, super-tasters).

\section{Results}

The present sample comprised 11 non-tasters, 22 tasters, and 15 super-tasters, which is consistent with the typical distribution found in other larger studies (Herz, 2011). Descriptive statistics are reported below in Table 1. Average pain tolerance time was $59 \mathrm{~s}(s d=62.50 ; 8-266 \mathrm{~s})$, which was consistently rated as being intense $(m=7.51, s d=1.91, S E M=.27)$ and unpleasant $(m=$ $7.77, s d=1.43, S E M=.21$ ). A moderate, positive correlation was observed between bitter taste intensity and pain intensity at pain tolerance, $r_{s}=.30, p=.04$. Adjusting the two outliers who experienced significant hypoalgesia (i.e., rating intensity at 1/10) did not impact on the strength of this association. Bitter intensity was not correlated with pain tolerance time $\left(r_{s}=.01, p=\right.$ $.95)$, unpleasantness at pain tolerance $\left(r_{s}=.09, p=.52\right)$, or fear of pain $\left(r_{s}=.22, p=.14\right)$ (see Fig. 1). 
Table 1 Medians (M) and $95 \%$ confidence intervals (CI) for PROP intensity ratings, CPT pain tolerance scores and FOP scores (Study 1)

\begin{tabular}{|c|c|c|c|c|c|c|}
\hline \multirow[b]{2}{*}{ Measurement } & \multicolumn{2}{|c|}{ Non-taster $(\mathrm{n}=11)$} & \multicolumn{2}{|c|}{ Taster $(\mathrm{n}=22)$} & \multicolumn{2}{|c|}{ Super-taster $(n=15)$} \\
\hline & M & {$[95 \% \mathrm{CI}]$} & M & {$[95 \% \mathrm{CI}]$} & M & {$[95 \% \mathrm{CI}]$} \\
\hline PROP & 5.50 & [1.6-8] & 28.40 & [18.4-33.6] & 61.60 & {$[52.8-76]$} \\
\hline FOP & 82.50 & [64-130] & 84.50 & {$[74-87]$} & 96.00 & [87-102] \\
\hline \multicolumn{7}{|l|}{ Pain tolerance } \\
\hline Time (s) & 36.00 & {$[21-60]$} & 45.90 & [24.8-92] & 36.00 & {$[24-56]$} \\
\hline Intensity & 7.20 & {$[5.6-8.5]$} & 7.85 & {$[7-8.1]$} & 8.40 & {$[8-9.3]$} \\
\hline Unpleasantness & 7.80 & {$[5.2-9.2]$} & 7.85 & {$[7-8.3]$} & 8.00 & {$[7.4-9.2]$} \\
\hline
\end{tabular}

PROP propylthiouracil , FOP Fear of Pain Questionnaire

The scatter plots revealed that non-tasters had greater variability in fear of pain compared with the tasters and supertasters. We further examined group differences in fear of pain with an independent samples Kruskal-Wallis test. There were significant group differences in fear of pain $[\mathrm{H}(2)=6.85, p=$ $.038, N=48]$, and a post-hoc Mann-Whitney $\mathrm{U}$ test revealed that this was specifically between the taster and super-taster groups, whereby the super-tasters had significantly higher fear of pain, $z(N=37)=-2.09, p=.036, r=.48$.

\section{Study two}

\section{Methods}

\section{Participants}

Forty participants volunteered, aged $20-42$ years $(M=24.35$, $S D=5.16)$, including $20(50 \%)$ women. Participants were recruited from university and social media (e.g., Facebook,
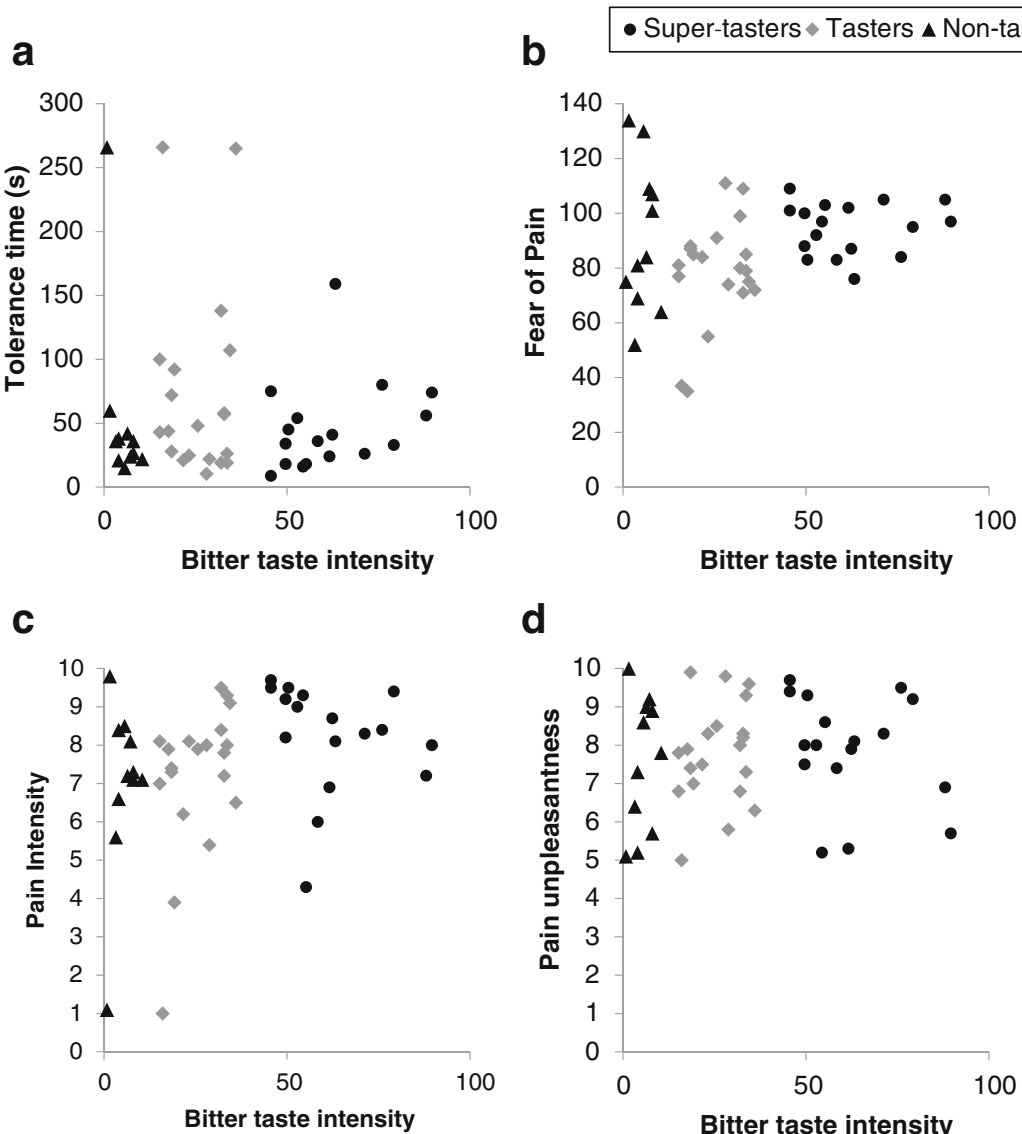

d

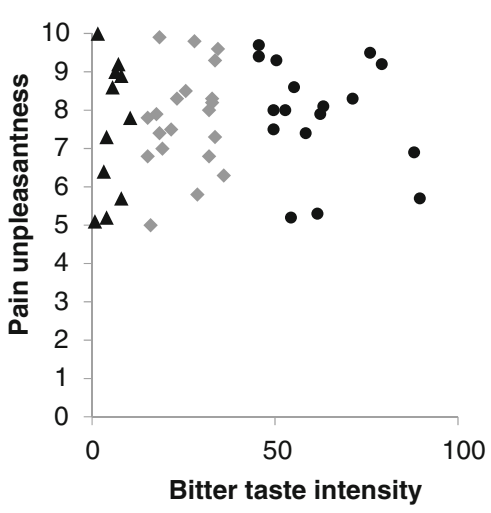

Fig. 1 Scatter plots of bitter intensity ratings from Study 1, where solid triangles indicate non-tasters, grey diamonds indicate tasters, and solid circles indicate super-tasters. a Pain tolerance time (ns); $\mathbf{b}$ fear of pain index (ns; however, Kruskal-Wallis test supported group differences

between tasters and super-tasters, with no differences with non-tasters); c pain intensity $(\mathrm{p}=.04)$; d pain unpleasantness $(\mathrm{ns})$. Note: all analyses were corrected for outliers 
Gumtree) advertisements. The inclusion criteria were the same as for Study 1.

\section{Materials and procedures}

To give a general indication of how a range of sensory information is processed, participants completed the Adolescent/ Adult Sensory Profile (Brown \& Dunn, 2002). This is a 60item scale that examines sensory sensitivities and preferences for everyday sensory experiences in relation to (1) tastes/ smells, (2) movement, (3) visual processing, (4) touch, (5) activity, and (6) auditory stimuli. Participants rate each item on a 5-point scale, from "Almost never" to "Always," with total scores ranging from 15 to 75 . Each item score is allocated to one of four overarching domains: low registration (frequently fail to perceive stimuli, or respond slowly to stimuli), sensation-seeking (pursue sensory stimuli), sensory sensitivity (distractibility, feeling discomfort with, or overwhelmed by, sensory stimuli), and sensation-avoiding (deliberate acts to prevent or reduce exposure to sensory stimuli). Sensitivity sub-scores were generated as the sum of sensitivity items for taste/smell (items 7 and 9, e.g., "I don't like strong tasting mints or candies"), touch (items 27, 31, 33, 34, e.g., "I'm uncomfortable wearing certain fabrics (e.g., wool, silk, tags)", "I dislike having my back rubbed"), and audition (items 51, 54,60 , e.g., "I startle easily at unexpected or loud noises"). The profile has sound internal consistency and convergent validity (Brown, Tollefson, Dunn, Cromwell, \& Filion, 2001).

\section{Thermal pain threshold, tolerance, and subjective experience}

Thermal stimuli were administered using a Medoc Pathway Pain and Sensory Evaluation System (Medoc Advanced Medical Systems, Israel). The Pathway system is specifically designed to administer objective, safe thermal experimental stimulation to humans. Stimulation was delivered through the thermal element on the Contact Heat-Evoked Potential Stimulator (CHEPS) thermode. The Pathway thermode was attached to the posterior forearm of the participant's non-dominant arm, approximately $5 \mathrm{~cm}$ proximal to the ulnar protuberance. To establish thermal pain threshold, the thermode was initially set at $32{ }^{\circ} \mathrm{C}$ and increased in temperature at a rate of $2{ }^{\circ} \mathrm{C} / \mathrm{s}$ using a staircase method, which is a valid and reliable method of assessing pain sensitivity (Gracely, Lota, Walter, \& Dubner, 1988). Participants were instructed to click a button when they perceived that the thermal stimulus had begun to feel painful (pain threshold). An interval of $60 \mathrm{~s}$ was allowed between trials in order to minimize the effects of sensitization. For all trials, the maximum temperature safety cut-off was set at $50^{\circ} \mathrm{C}$.

Participants were then asked to rate the subjective intensity and unpleasantness of stimuli that were innocuous $\left(33^{\circ} \mathrm{C}\right)$, moderately painful $\left(41^{\circ} \mathrm{C}\right)$, and of high intensity $\left(44^{\circ} \mathrm{C}\right)$, with a total of 42 trials at each temperature delivered across two blocks. The thermode was removed from the arm between each block to give the participant a rest. In each trial, the participant was presented with a fixation cross $(1 \mathrm{~s})$, a cue (colored flames: $33{ }^{\circ} \mathrm{C}=$ green flame, $41{ }^{\circ} \mathrm{C}=$ orange flame, $44^{\circ} \mathrm{C}=$ red flame), and the $4-\mathrm{s}$ stimulus (including $1 \mathrm{~s}$ to reach the target temperature and $1 \mathrm{~s}$ to return to baseline). Participants were given $3 \mathrm{~s}$ to rate the intensity and unpleasantness of the stimulus $(0=$ no pain/not unpleasant; $10=$ extreme pain/extremely unpleasant).

\section{Data analysis}

Data were analyzed with SPSS, version 20. The subscales of the Adult/Adolescent Sensory Profile were normally distributed and had no outliers. Average intensity/unpleasantness ratings were generated for each stimulus intensity. The association between the Adult/Adolescent Sensory Profile subscales, pain threshold, and intensity/unpleasantness ratings were examined using Pearson correlations.

Canonical correlation was then used to examine the association between sensory sensitivity for specific modalities (touch, audition, taste/smell) and thermal pain intensity ratings (innocuous, moderate, high). Rather than examining the association between individual variables, canonical correlation estimates canonical variates, which are the linear combination of variables comprised in the set of independent and dependent variables. The canonical variate reflects the relative linear relationship between independent and dependent variables. The significance of the model is indicated through the F-statistic, the strength of the association is determined through the percentage variance explained and the Redundancy Index. The Redundancy Index is a calculation of the variance shared between each independent or dependent variable and the variate multiplied by the total variance explained by the variate (i.e., the Canonical root). A higher redundancy index is desirable as this shows that a high proportion of variance in the DVs is explained by the IVs, and vice versa. The canonical crossloadings (i.e., standardized correlation weight multiplied by the canonical correlation) are then used to determine the relative contribution of each variable to the variate. Sensitivity analyses were undertaken to determine whether any specific variable had greater impact on the results. The data met the assumptions for canonical correlation with linearity and low multicollinearity (i.e., preliminary linear regression analyses showed that the tolerance inflation factors were all $>.2$, and the variance inflation factors were $<10$ ).

\section{Results}

Average pain threshold was $38^{\circ} \mathrm{C}(\mathrm{SD}=3.30,95 \%$ CI: $37.7-$ 39.8 ), and, on average, participants scored within the normal ranges for sensation seeking $(m=48.75, s d=7.39)$, sensory sensitivity $(m=36.58, s d=5.10)$, sensation avoiding $(m=$ 
36.42, $s d=6.33)$, and low registration $(m=33.53, s d=6.69)$. Only sensory sensitivity was significantly and highly correlated with both intensity $(r=.51, p=.002)$ and unpleasantness $(r$ $=.48, p=.003$ ) of the high intensity stimulus. Descriptive statistics are reported in Table 2 and Fig. 2. Sensation avoiding, seeking, and low registration did not show clear relationships with pain sensitivity or ratings of intensity and unpleasantness.

A canonical correlation was undertaken to specifically examine the association between sensory sensitivity for three key sensory modalities (touch, audition, taste/smell) and intensity ratings of the three thermal stimuli (innocuous, $33{ }^{\circ} \mathrm{C}$; moderate, $41{ }^{\circ} \mathrm{C}$; high $44{ }^{\circ} \mathrm{C}$ ). This revealed one significant canonical variate pair $(p<.01)$, which explained $32.7 \%$ of the variance in the sensory sensitivities, and $10.3 \%$ of variance in intensity ratings. Table 3 displays the canonical correlations, canonical cross-loadings, shared variance and redundancy indices for the full model, and the sensitivity analyses whereby the independent and dependent variables were sequentially omitted. The sensitivity analyses showed that there was no marked impact on shared variance and redundancy index with omission of ratings of the $33{ }^{\circ} \mathrm{C}$ stimulus; however, there was no significant association upon omission of the moderate $\left(41{ }^{\circ} \mathrm{C}\right)$ and high $\left(44{ }^{\circ} \mathrm{C}\right)$ stimulus ratings, as well as omission of the touch- and audition-specific sensitivities. That is, the sensitivity analyses suggest that there was only a significant association for two sensory modalities (touch, audition) and moderate-high thermal stimuli. Self reported sensitivity to taste/smell was not associated with pain sensitivity (Fig. 3).

Table 2 Correlation coefficients between pain (threshold, intensity, and unpleasantness) ratings at each thermal stimulus intensity, and the four $\mathrm{A} /$ ASP domains (Study 2)

\begin{tabular}{lllll}
\hline \multicolumn{5}{c}{ Correlation co-efficient } \\
\cline { 2 - 5 } & $\begin{array}{l}\text { Sensation } \\
\text { seeking }\end{array}$ & $\begin{array}{l}\text { Sensory } \\
\text { sensitivity }\end{array}$ & $\begin{array}{l}\text { Sensory } \\
\text { avoiding }\end{array}$ & $\begin{array}{l}\text { Low } \\
\text { registration }\end{array}$ \\
\hline Pain threshold & .08 & .10 & -.06 & .04 \\
Intensity & & & & \\
Innocuous & .06 & .20 & -.05 & .25 \\
Moderate & .07 & .26 & -.02 & .28 \\
High & -.12 & $.51^{* *}$ & .05 & .13 \\
Unpleasantness & & & & \\
Innocuous & -.15 & .20 & .31 & .09 \\
Moderate & -.07 & .29 & $.34 *$ & .16 \\
High & -.10 & $.48^{* * *}$ & .04 & .13 \\
\hline
\end{tabular}

Note: $\mathrm{N}=40$, Bonferroni correction, $\alpha=.002$

$* \mathrm{p}=.04$, trend only

$* * \mathrm{p}=.002$

$* * * \mathrm{p}=.003$

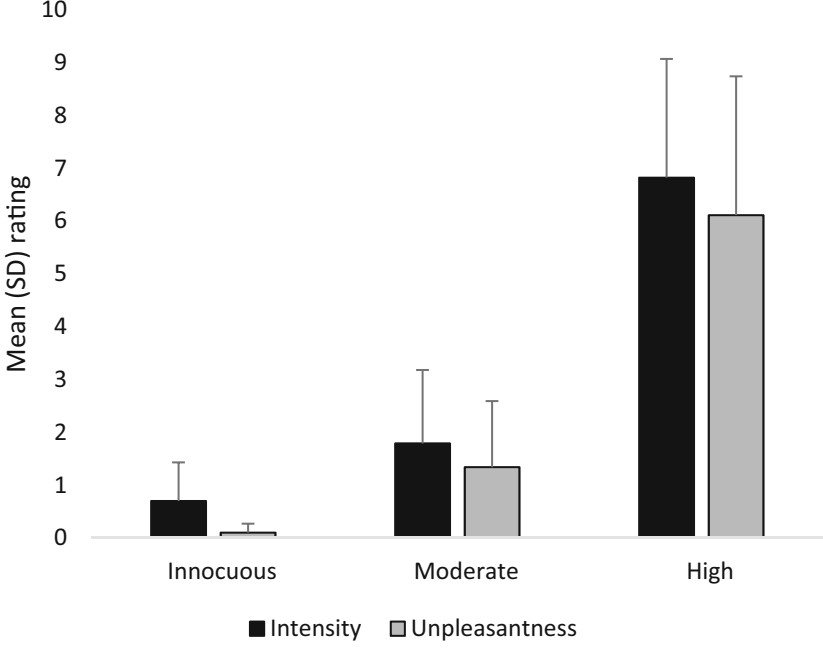

Fig. 2 Pain intensity and unpleasantness ratings of the innocuous (33 ${ }^{\circ} \mathrm{C}$ ), moderate $\left(41^{\circ} \mathrm{C}\right)$, and high $\left(44^{\circ} \mathrm{C}\right)$ thermal stimuli in Study 2

\section{General discussion}

We undertook two studies that investigated whether increased sensitivity in one sensory modality (i.e., taste, audition, touch) is associated with increased sensitivity in another (i.e., pain). The first study specifically examined whether bitter taste intensity was associated with enhanced sensitivity to pain (i.e., pain tolerance, and ratings of intensity and unpleasantness at the point of tolerance), and fear of pain, in a sample of healthy young adults. We found that bitter taste sensitivity was positively associated with perceiving pain at the point of tolerance to be more intense. However, there was no relationship between bitter taste sensitivity and pain tolerance time. These effects suggest that persons who are prone to experiencing sensations (whether specific to taste or nociception) as more intense respond behaviorally in a similar manner to persons who do not report these sensitivities. Participants with greater sensitivity to bitter taste (i.e., super-tasters) were significantly more fearful of pain than those who were tasters, suggesting that having a suprathreshold bitter taste sensitivity was tied to being more afraid of pain.

The second study examined the association between thermal pain sensitivity - specifically pain threshold, and intensity and unpleasantness ratings of innocuous $\left(33^{\circ} \mathrm{C}\right)$, moderate $\left(41{ }^{\circ} \mathrm{C}\right)$, and high $\left(44^{\circ} \mathrm{C}\right)$ intensity thermal stimuli - and sensitivity to other sensory modalities (especially taste/smell, touch and auditory). We expected thermal pain sensitivity would be related to cross-modality sensory sensitivity and the tendency to avoid sensations across modalities. We found that participants who had higher sensitivity to these exteroceptive inputs in general also rated the most noxious thermal stimulus $\left(44^{\circ} \mathrm{C}\right)$ to be more intense and unpleasant, indicating that they were generally more likely to be overwhelmed by sensations across modalities. The tendency to avoid sensations was only modestly associated with perceived 
Table 3 Sensitivity analysis to determine the impact of specific independent and dependent variables on the canonical correlation (Study 2)

Results after deletion of:

\begin{tabular}{|c|c|c|c|c|c|c|c|}
\hline & \multirow[b]{2}{*}{ All variables } & \\
\hline & & $33^{\circ} \mathrm{C}$ & $41^{\circ} \mathrm{C}$ & $44^{\circ} \mathrm{C}$ & Touch & Audition & Taste/Smell \\
\hline Canonical correlation $(\mathrm{R})$ & .56 & .52 & .55 & .47 & .51 & .50 & .53 \\
\hline Canonical root $\left(\mathrm{R}^{2}\right)$ & .32 & .27 & .31 & .22 & .26 & .25 & .28 \\
\hline \multicolumn{8}{|l|}{ Independent variate } \\
\hline \multicolumn{8}{|l|}{ Canonical cross-loadings } \\
\hline Innocuous & .44 & Omitted & - & - & - & .20 & - \\
\hline Moderate & -.30 & .25 & Omitted & - & - & .08 & - \\
\hline High & .53 & .35 & - & Omitted & - & .38 & - \\
\hline Shared variance & .46 & .49 & ns & ns & ns & ns & .62 \\
\hline Redundancy & .15 & .14 & ns & ns & ns & ns & .17 \\
\hline \multicolumn{8}{|l|}{ Dependent variate } \\
\hline \multicolumn{8}{|l|}{ Canonical cross-loadings } \\
\hline Touch & .37 & .46 & - & - & Omitted & - & .44 \\
\hline Audition & .37 & .46 & - & - & - & Omitted & .44 \\
\hline Taste & .25 & .081 & - & - & - & - & Omitted \\
\hline Shared variance & .33 & .36 & ns & ns & ns & ns & .38 \\
\hline Redundancy & .10 & .099 & $\mathrm{~ns}$ & ns & ns & ns & .11 \\
\hline
\end{tabular}

unpleasantness of the moderate intensity thermal stimulus $\left(41^{\circ} \mathrm{C}\right)$, but not with ratings of the innocuous or high $\left(44{ }^{\circ} \mathrm{C}\right)$ intensity painful stimuli. Finally, we found that selfreported sensitivity to taste/smell stimuli was not associated with sensitivity to thermal stimuli. Rather, only sensitivity to touch and auditory stimuli were associated with sensitivity to moderate-high intensity thermal stimuli. In line with the results from Study 1, although cross-modal sensory sensitivity does indeed seem to occur for suprathreshold noxious experiences, avoidance tendencies do not show a consistent association with the subjective experience of acute pain in healthy young adults. Collectively, these findings suggest that heightened sensory sensitivity to painful stimuli may in fact generalize to other sensory domains, especially auditory and touch sensations.

It should be noted that the associations that we observed between bitter taste sensitivity and pain sensitivity were not as strong as those observed in clinical samples (Erden et al., 2007). In fact, we found no consistent evidence for an association between self-reported taste/smell sensitivity and pain sensitivity. This suggests that the strength of cross-modal sensitivities in those with chronic pain may be driven mechanisms such as central sensitization, whereby there is an imbalance in excitatory and inhibitory signals, resulting in hyperalgesia in the injured area, inflammation, and spreading of pain to uninjured body areas (Kwon, Altin, Duenas \& Alev, 2013). We suggest that in the context of chronic pain, sensitization may also lead to greater excitability, and diminished inhibition, of other salient sensory inputs, such as those pertaining to taste. Moreover, the crossmodal sensitivities may be more cognitive in nature, particularly from heightened fear of unpleasant and painful sensations (Formaker \& Frank, 2000; Small, 2010).

Our findings reveal that healthy young adults with heightened sensitivity to bitter taste find intolerable pain to be more intense, but not necessarily more unpleasant. This finding is unusual given that participants were instructed to keep their arm immersed until it was intolerable, rather than for a set period of time. Ideally when administered in this way, all persons will withdraw their hand when they reach the same approximate level of subjective intensity, usually around 7$10 / 10$. As such, our findings seem to indicate that persons who are more sensitive to noxious or unpleasant sensations (i.e., super-tasters) may have learned to tolerate sensations that are more intense, which leads to a similar behavioral outcome (i.e., pain tolerance time). While super-tasters were more fearful of pain than tasters in Study 1, the tendency to avoid sensations was not associated with altered heat pain experience (threshold, intensity or unpleasantness) in Study 2.

There was a stronger association between pain sensitivity and sensitivity to touch and auditory stimuli. This suggests that enhanced cross-modal sensitivities in healthy young adults seem to be specific to somatosensory sensations (i.e., cutaneous touch, such as disliking being stroked or being touched by certain fabrics) and the sensitivity to salient environmental sensory cues (i.e., auditory, such as enhanced startle responses to loud sounds). The likely consequences of such sensory preferences and sensitivities cannot be determined from the present study; however, it is clear that understanding one's sensory preferences is useful in clinical and occupational settings. For instance, persons with low sensitivity and low avoidance may 
a

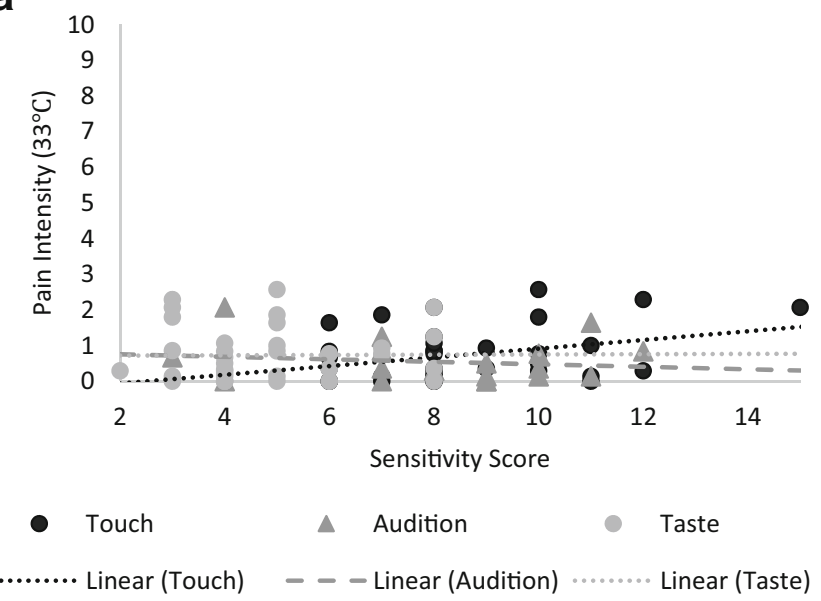

b

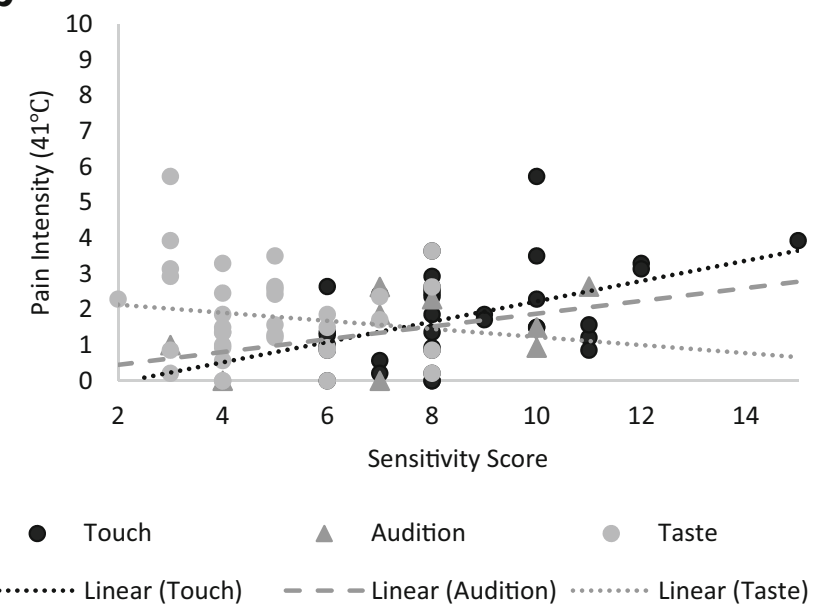

C

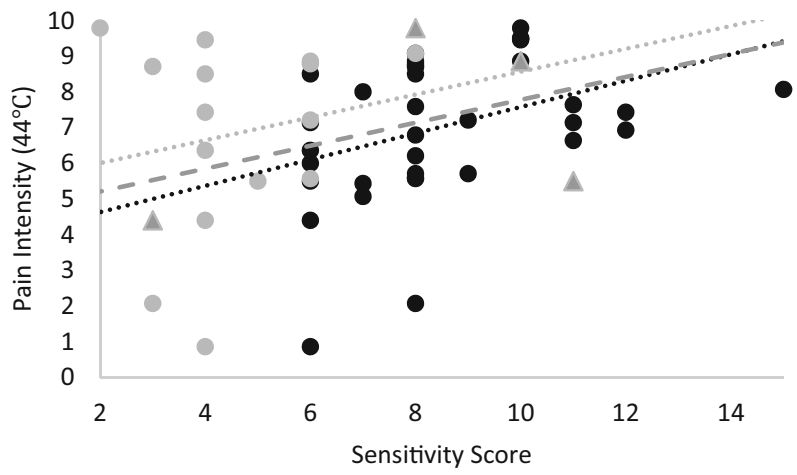

$\begin{array}{cccc}- \text { Touch } & \mathbf{4} \text { Audition } & \text { Taste } \\ \ldots . . . . & \text { Linear (Touch) } & - & -\end{array}$

Fig. 3 Scatterplots between sensory sensitivity scores for touch (enclosed black circles), audition (grey triangles), and taste/smell (grey circles) modalities and ratings of each thermal pain stimulus: a innocuous, $33{ }^{\circ} \mathrm{C}$; b moderate $41{ }^{\circ} \mathrm{C}$; $\mathbf{c}$ high, $44{ }^{\circ} \mathrm{C}$ (Study 2)

benefit from high levels of sensory enrichment, whereas persons with high sensitivity and avoidance would benefit more when sensory inputs are highly controlled (e.g., dim lighting, soft music). Given the present findings, these considerations may be especially pertinent in the context of acute pain (e.g., after injury or surgery) in order to minimize potential negative effects of sensory stimulation on pain experience.

The limitations of this study should be considered when drawing generalizations. First, the sample population for both studies was healthy, young, and pain-free. It may be that increases in cross-modal sensitivities and avoidance tendencies only arise in the context of chronic pain, with central sensitization and associated neuroplasticity to the neural pathways processing pain over time (Wand et al., 2011). In such a healthy population, both bitter taste sensitivity and pain sensitivity evidently have high levels of variability, resulting in fairly small effect sizes. The significant association between bitter taste sensitivity and intensity at the point of pain tolerance was only marginal after correcting for multiple comparisons.

The findings from both studies must be considered in the context of the respective study designs. Study 1 was respondent-centred (i.e., subjective perception of intolerability), and Study 2 was predominantly stimulus-centred (i.e., subjective perception of intensity and unpleasantness at standard stimulus intensities). Although unpleasantness of intolerable pain was not correlated with bitter taste intensity in Study 1 (as one might expect, given that the point of pain tolerance should be at the maximum subjective point of intensity and unpleasantness), unpleasantness ratings of a standard moderately or highly painful thermal stimuli were positively correlated with self-reported sensory sensitivity in Study 2 . Therefore using a respondent-centred experimental task did not reveal consistent cross-modal associations between taste and pain sensitivity, whereas a stimulus-centred experimental task did reveal significant associations between touch, auditory, and pain sensitivity (but not taste/smell) when the thermal stimulus was moderate-high intensity.

In conclusion, the present studies found that in a healthy sample of young adults, bitter taste sensitivity was positively associated with intensity of pain at the point at which it can no longer be tolerated, with super-tasters showing higher fear of pain than tasters. Additionally, higher perception of unpleasantness and intensity of painful thermal heat was found to be related to higher sensory sensitivity across exteroceptive modalities, especially those relating to somatosensation and environmental orienting (i.e., audition). Considering this is the first study to examine these relationships in a healthy sample, further research is required to comprehensively understand the extent of cross-modal sensitivities in both clinical and non-clinical populations. In particular, confirmation of the genetic PROP taster status, and evaluation of sensitivity across sensory modalities, in persons with and without chronic pain is warranted to confirm and extend the present findings. 
Acknowledgments MJG was supported by a National Health and Medical Research Council (NHMRC, Australia) early career fellowship (APP1036124). The authors acknowledge Ziarih Hawi, who assisted in the preparation of the PROP filter papers.

\section{Compliance with ethical standards}

Conflicts of interest and disclosures The authors have no financial or other relationships that might lead to a conflict of interest. This manuscript is not currently under consideration for publication elsewhere. Informed consent was obtained from all participants, and the rights of the subjects were protected.

\section{References}

Bartoshuk, L. M., Duffy, V. B., \& Miller, I. J. (1994). PTC/PROP tasting: Anatomy, psychophysics, and sex effects. Physiology \& Behavior, 56(6), 1165-1171. doi:10.1016/0031-9384(94)90361-1

Bastian, B., Jetten, J., \& Hornsey, M. J. (2014). Gustatory pleasure and pain. The offset of acute physical pain enhances responsiveness to taste. Appetite, 72, 150-155. doi:10.1016/j.appet.2013.10.011

Brown, C., \& Dunn, W. (2002). Adolescent/ Adult Sensory Profile [Measurement instrument]. Retrieved from http://www. pearsonclinical.com/therapy/products/100000434/adolescentadultsensory-profile.html

Brown, C., Tollefson, N., Dunn, W., Cromwell, R., \& Filion, D. (2001). The adult sensory profile: Measuring patterns of sensory processing. The American Journal of Occupational Therapy, 55(1), 75-82.

Duffy, V. B., Davidson, A. C., Kidd, J. R., Kidd, K. K., Speed, W. C., Pakstis, A. J., \& Bartoshuk, L. M. (2004). Bitter receptor gene (TAS2R38), 6-n-propylthiouracil (PROP) bitterness and alcohol intake. Alcoholism: Clinical and Experimental Research, 28(11), 1629-1637. doi:10.1097/01. ALC.0000145789.55183.D4

Eggleston, K., White, T. L., \& Sheehe, P. R. (2010). Adding cocoa to sucrose: The effect on cold pain tolerance. Chemical Senses, 35(4), 269-277. doi:10.1093/chemse/bjq016

Erden V, Basaranoglu G, Korkut Y, Delatioglu H, Yangin Z, Kiroglu S (2007). Relation between bitter taste sensitivity and incidence or intensity of propofol injection pain. European Journal of Anaesthesiology, 24(6),516-20.

Formaker, B. K., \& Frank, M. E. (2000). Taste function in patients with oral burning. Chemical Senses, 25, 575-581. doi:10.1093 /chemse/25.5.575

Gracely RH, Lota L, Walter DJ, Dubner R. (1988). A multiple random staircase method of psychophysical pain assessment. Pain, 32(1), $55-63$.

Green BG, Dalton P, Cowart B, Shaffer G, Rankin K, Higgins J. (1996). Evaluating the 'Labeled Magnitude Scale' for measuring sensations of taste and smell. Chemical Senses 21, 323-34.

Herbert, C., Platte, P., Wiemer, J., Macht, M., \& Blumenthal, T. D. (2014). Supertaster, super reactive: Oral sensitivity for bitter taste modulates emotional approach and avoidance behaviour in the affective startle paradigm. Physiology \& Behavior, 135, 198-207. doi:10.1016/j.physbeh.2014.06.002

Herz, R. S. (2011). PROP taste sensitivity is related to visceral but not moral disgust. Chemosensory Perception, 4(3), 72-79. doi:10.1007 /s12078-011-9089-1

Johnston, C. C., Fernandes, A. M., \& Campbell-Yeo, M. (2011). Pain in neonates is different. Pain, 152, S65-S73. doi:10.1016/j. pain.2010.10.008

Koenig, J., Jarczok, M. N., Ellis, R. J., Bach, C., Thayer, J. F., \& Hillecke, T. K. (2014). Two-week test-retest stability of the cold pressor task procedure at two different temperatures as a measure of pain threshold and tolerance. Pain Practice, 14(3), E126-E135. doi:10.1111 /papr.12142

Kulkarni, B., Bentley, D. E., Elliott, R., Youell, P., Watson, A., Derbyshire, S. W. G., \& Jones, A. K. P. (2005). Attention to pain localization and unpleasantness discriminates the functions of the medial and lateral pain systems. European Journal of Neuroscience, 21(11), 3133-3142. doi:10.1111/j.14609568.2005.04098.x

Kwon, M., Altin, M., Duenas, H., \& Alev, L. (2013). The role of descending inhibitory pathways on chronic pain modulation and clinical implications. Pain Practice. doi:10.1111/papr.12145

Macht, M., \& Mueller, J. (2007). Increased negative emotional responses in PROP supertasters. Physiology and Behaviour, 90, 466-472. doi:10.1016/j.physbeh.2006.10.011

McNeil, D. W., \& Rainwater, A. J. (1998). Development of the fear of pain questionnaire-III. Journal of Behavioral Medicine, 21(4), 389 410. doi:10.1023/A:101878283121

Priya, S. A., Siddanagoudar, S., Nalluwar, S. C., \& Neelam, D. (2015). Correlation of pain sensitivity and sweet taste in healthy male adults. National Journal of Physiology, Pharmacy and Pharmacology, 5, 25-27. doi:10.5455/njppp.2015.5.110720141

Roelofs, J., Peters, M. L., Deutz, J., Spijker, C., \& Vlaeyen, J. W. S. (2005). The Fear of Pain Questionnaire (FPQ): Further psychometric examination in a non-clinical sample. Pain, 116(3), 339-346. doi:10.1016/j.pain.2005.05.003

Small, D. M. (2010). Taste representation in the human insula. Brain Structure and Function, 214, 551-561. doi:10.1007/s00429-0100266-9

Small, D. M., \& Apkarian, A. V. (2006). Increased taste intensity perception exhibited by patients with chronic back pain. Pain, 120(1-2), 124-130. doi:10.1016/j.pain.2005.10.021

Tabachnick, B. G., \& Fidell, L. S. (2013). Using multivariate statistics (6th ed.). Boston, MA: Pearson International Ed.

Vogt, B. A. (2005). Pain and emotion interactions in subregions of the cingulate gyrus. Nature Reviews Neuroscience, 6(7), 533-544. doi:10.1038/nrn1704

Wand, B. M., Parkitny, L., O’Connell, N. E., Luomajoki, H., McAuley, J. H., Thacker, M., \& Moseley, G. L. (2011). Cortical changes in chronic low back pain: Current state of the art and implications for clinical practice. Manual Therapy, 16(1), 15-20. doi:10.1016/j. math.2010.06.008

Wolff, B. B. (1984). Methods of testing pain mechanisms in normal man. In P. D. Wall \& R. Melzack (Eds.), Textbook of pain (pp. 186-194). Edinburgh: Churchill Livingstone. 